\title{
Magnetic Impurities, Integrable Vortices and the Toda Equation
}

\author{
Sven Bjarke Gudnason ${ }^{1}$, Calum Ross ${ }^{2,3}$ \\ ${ }^{1}$ Institute of Contemporary Mathematics, School of Mathematics and Statistics, Henan University, \\ Kaifeng, Henan 475004, P. R. China \\ ${ }^{2}$ Department of Physics, University College Cork, Cork T12 K8AF, Ireland \\ ${ }^{3}$ Department of Physics and Research and Education Center for Natural Sciences, Keio \\ University, Hiyoshi 4-1-1, Yokohama, Kanagawa 223-8521, Japan \\ E-mail: gudnason(at)henu.edu.cn, c.ross (at)keio.jp
}

\begin{abstract}
The five integrable vortex equations, recently studied by Manton, are generalized to include magnetic impurities of the Tong-Wong type. Under certain conditions these generalizations remain integrable. We further set up a gauge theory with a product gauge group, two complex scalar fields and a general charge matrix. The second species of vortices, when frozen, are interpreted as the magnetic impurity for all five vortex equations. We then give a geometric compatibility condition, which enables us to remove the constant term in all the equations. This is similar to the reduction from the Taubes equation to the Liouville equation. We further find a family of charge matrices that turn the five vortex equations into either the Toda equation or the Toda equation with the opposite sign. We find exact analytic solutions in all cases and the solution with the opposite sign appears to be new.
\end{abstract}

KEywords: Vortex Equations, Integrable Vortices, Toda Equation, Field Theory on Curved Spaces, Impurities in Field Theory 


\section{Contents}

1 Introduction $\quad 1$

1.1 Conventions for local geometry 3

2 Exotic vortex equations 3

3 Magnetic impurities $\quad 5$

$\begin{array}{lll}3.1 & \text { Exotic Liouville-type equations with impurities } & 6\end{array}$

4 Product gauge groups $\quad 8$

4.1 Toda system 11

$\begin{array}{lll}4.2 & \text { Impurities from product gauge groups } & 13\end{array}$

4.2.1 What became of the topological charge? 15

$\begin{array}{lll}4.3 \text { General coupled vortex equations } & 15\end{array}$

$\begin{array}{llr}5 & \text { Discussion and conclusion } & 16\end{array}$

\section{Introduction}

Vortex equations have been studied in many fields of physics ever since they made their appearance in the Ginzburg-Landau equations describing magnetic fluxes penetrating a type-II superconductor, giving rise to the Abrikosov lattice, see e.g. [1-4]. Other vortex equations have been discovered, like the Jackiw-Pi vortices in nonrelativistic Chern-Simons theory [5, 6], the Ambjørn-Olesen vortices in the electroweak theory [7] and Popov vortices in the reduction a lá Witten [8] of self-dual Yang-Mills theory to the 2-sphere [9] instead of the hyperbolic plane [8]. All of the above vortex equations were put on equal footing by Manton in Ref. [10] and an extra equation was included, dubbed the Bradlow vortex equation because of its resemblance to the Taubes vortex equation in the Bradlow limit. These "exotic" vortex equations are all integrable in two dimensions, but not all on the same base Riemann surface. It turns out that there is a direct connection between the signs (or "0") of the Fayet-Iliopoulos parameter (the constant term) of the vortex equation and the (constant) curvature of the Riemann surface on which they are integrable. The coefficient in front of the vortex field has the same property, not related to the base Riemann surface, but to the constant curvature of the Baptista surface [11]. Witten showed in the seminal paper that the integrable version of the Taubes equation, which on the hyperbolic plane becomes the Liouville equation, has the interpretation as a suitable reduction of integrable 
instantons in $\mathbb{R}^{4}$ to a sphere and a hyperbolic plane [8]. Doing the opposite reduction, one arrives at the Popov vortex equations, which are integrable on a 2-sphere [9]. Finally, such a reduction was generalized to all the exotic vortex equations by Contatto and Dunajski $[12]$.

Impurities have recently become an active area of theoretical research, perhaps starting with the elegant interpretation given to magnetic impurities in the Taubes equation by Tong and Wong, as a second "frozen" vortex species [13]. Impurities in integrable hyperbolic (Taubes) vortices have been studied by Cockburn-Krusch-Muhamed [14], where the interpretation of a delta-function impurity as a source of extra vortices is made. Impurities have further made possible the discovery of spectral walls in the dynamics of kinks in $1+1$ dimensions [15].

In this paper we generalize all five exotic vortex equations to include an impurity field, and discuss when they remain integrable. Then we consider a $\mathrm{U}(1)^{2}$ gauge theory with two complex scalar (Higgs) fields and a general charge matrix, giving rise to a nontrivial mixing of the vortex equations, following Ref. [16]. We generalize this theory to account for all five vortex equations. We find a geometric compatibility condition, for which we can remove the Fayet-Iliopoulos (constant) term from the entire system of equations. This puts a constraint on the charge matrix as well as the Fayet-Iliopoulos (constant) parameters. Choosing a special family of charge matrices, we reduce the system of equations to the integrable Toda system, for the case of a specific sign in the vortex equations. We further generalize the solution of Kostant-Leznov-Saveliev (KLS) [17-19] to both signs of the constant and explain the trivial case of coupled Bradlow equations. This establishes exact analytic solutions, in closed form, for the Taubes-Toda, Popov-Toda, Jackiw-Pi-Toda, AmbjørnOlesen-Toda and Bradlow-Toda vortices. The Taubes-Toda equation has the opposite sign to the normal Toda equation and the KLS solution is not directly applicable. We find a generalization of the KLS solution to the Toda equation of the opposite sign, which to the best of our knowledge is new. Interestingly, the normal Toda equation corresponds naturally to the coupled Popov, coupled Jackiw-Pi and coupled Ambjørn-Olesen equations on certain background manifolds. Finally, we generalize the frozen vortex explanation for impurities of Tong and Wong to all five exotic vortex equations.

The paper is organized as follows. In Sec. 2, we review the five exotic vortex equations. In Sec. 3, we generalize the exotic vortex equations to include magnetic impurities. In Sec. 4, we set up the gauge theory with product gauge groups for exotic vortices with a generic charge matrix and find the geometric compatibility condition. In Sec. 4.1 we find the solution for the charge matrix that reduces the coupled vortex equations to integrable Toda equations. In Sec. 4.2, we use the theory to rederive the magnetic impurity as a frozen second species of vortex. In Sec. 4.3, we consider some examples of nonintegrable vortex equations and give an example of a numerical solution. Finally, we conclude the paper with a discussion in Sec. 5. Before starting off, we will quickly set the conventions used in the rest of the paper. 


\subsection{Conventions for local geometry}

It will prove convenient to set the notation and conventions for the paper. For a Riemann surface $M_{0}$ with constant Gauss curvature $K_{0}$ we work in terms of a local complex coordinate, $z$. The Riemann surface ${ }^{1}$ has the metric

$$
\mathrm{d} s^{2}=\Omega_{0} \mathrm{~d} z \mathrm{~d} \bar{z}=\frac{4}{\left(1+K_{0}|z|^{2}\right)^{2}} \mathrm{~d} z \mathrm{~d} \bar{z}
$$

which admits the local complexified frame

$$
e=\frac{2 \mathrm{~d} z}{1+K_{0}|z|^{2}}
$$

In terms of the frame field, the structure and Gauss equations are

$$
\begin{aligned}
\mathrm{d} e-\mathrm{i} e & \wedge \Gamma=0, \\
\mathrm{~d} \Gamma=\mathcal{R} & =\frac{\mathrm{i}}{2} K_{0} e \wedge \bar{e},
\end{aligned}
$$

where

$$
\Gamma=\mathrm{i} K_{0} \frac{z \mathrm{~d} \bar{z}-\bar{z} \mathrm{~d} z}{1+K_{0}|z|^{2}}
$$

is the spin connection and $\mathcal{R}$ is the curvature 2 -form.

\section{Exotic vortex equations}

In Ref. [10] a generalization of the standard hyperbolic vortex equations was introduced. These vortices are pairs $(\phi, A)$ of a connection $A$ and a smooth section $\phi$ on a line bundle over a Riemann surface, $M_{0}$. In the case that $M_{0}$ is non-compact, appropriate asymptotics, $|\phi| \rightarrow 1$ on $\partial M_{0}$, need to be imposed so that the energy is finite $[2,3]$. These exotic vortex equations are

$$
(\mathrm{d} \phi-\mathrm{i} A \phi) \wedge e=0, \quad F=\mathrm{d} A=\left(\lambda_{0}-\lambda|\phi|^{2}\right) \omega_{0},
$$

where $\omega_{0}$ is the Kähler form on $M_{0}$.

In terms of a local complex coordinate $z$ on $M_{0}$ these become

$$
\begin{aligned}
\partial_{\bar{z}} \phi-\mathrm{i} A_{\bar{z}} \phi & =0 \\
F_{z \bar{z}}=\partial_{z} A_{\bar{z}}-\partial_{\bar{z}} A_{z} & =\frac{\mathrm{i}}{2} \Omega_{0}\left(\lambda_{0}-\lambda|\phi|^{2}\right) .
\end{aligned}
$$

By decomposing the Higgs field as $\phi=e^{h+i \chi}$ a generalization of the Taubes equation is arrived at

$$
-\frac{4}{\Omega_{0}} \partial_{z} \partial_{\bar{z}} h=\left(\lambda_{0}-\lambda e^{2 h}\right)-\frac{2 \pi}{\Omega_{0}} \sum_{r=1}^{N} \delta\left(z-Z_{r}\right),
$$

\footnotetext{
${ }^{1}$ Here $M_{0}$ will either be $\mathbb{S}^{2}, \mathbb{H}^{2}$ or $\mathbb{R}^{2}$. In the two latter cases $z$ is a global coordinate, whereas for $\mathbb{S}^{2}$ we need at least two patches to cover the space globally.
} 
where $\Omega_{0}$ is the conformal factor relating the metric on $M_{0}$ to the flat metric on $\mathbb{R}^{2}$ and $Z_{r} \in \mathbb{C}$ are the positions of $N$, not necessarily separated, vortices.

A scaling argument given in Ref. [10] allows us to restrict $\lambda_{0}$ and $\lambda$ to take the values $-1,0,1$ giving nine possible equations. By integrating this equation, and imposing that the left-hand side be positive, as it is the integral of the magnetic field corresponding to the magnetic flux, allows us to exclude four cases. This leaves the five cases:

\begin{tabular}{llrr} 
& Vortices & $\lambda_{0}$ & $\lambda$ \\
\hline 1. & Hyperbolic vortices & 1 & 1 \\
2. & Popov vortices & -1 & -1 \\
3. & Jackiw-Pi vortices & 0 & -1 \\
4. & Ambjørn-Olesen vortices & 1 & -1 \\
5. & Bradlow vortices & 1 & 0
\end{tabular}

We refer to these as the $\left(\lambda_{0}, \lambda\right)$ vortex equations. As was observed in Ref. [10] and we will review below, these equations are integrable on a constant curvature Riemann surface with Gauss curvature $K_{0}=-\lambda_{0}$.

Hyperbolic vortices have been well studied, many of the details are given in Ref. [2], and are integrable on 2-dimensional hyperbolic space, $\mathbb{H}^{2}$. Most commonly the Poincaré disk model of $\mathbb{H}^{2}$ (here we take the unit disk) is used. On the disk a hyperbolic vortex is given by a holomorphic map

$$
f: \mathbb{H}^{2} \rightarrow \mathbb{H}^{2},
$$

such that $|f| \rightarrow 1$ as $|z| \rightarrow 1$. This means that $f$ can be represented as a finite Blaschke product as was first established in the seminal paper [8].

The Popov vortex equations were introduced in Ref. [9] as a dimensional reduction of $\mathrm{SU}(1,1)$ instantons. These equations on $\mathbb{S}^{2}$ were further considered in Ref. [20] where solutions were expressed in terms of rational maps

$$
R: \mathbb{S}^{2} \rightarrow \mathbb{S}^{2}
$$

Bradlow vortex equations, first introduced in Ref. [10] have exact solutions on $\mathbb{H}^{2}$, but due to the simplicity of the vortex equation it does in fact have integrable solutions on many compact domains of various curvatures, including vanishing curvature [21].

More generally, we call the $\left(\lambda_{0}, \lambda\right)$ vortex equations integrable when they can be solved by a holomorphic map between two constant curvature Riemann surfaces,

$$
f: M_{0} \rightarrow M
$$

where $M_{0}$ has Gauss curvature $K_{0}=-\lambda_{0}$ and $M$ has Gauss curvature $K=-\lambda$ (away from ramification points of $f$ that correspond to the zeros of $\phi$ ). In fact, a general expression for the norm-squared of the Higgs field is [10]

$$
|\phi|^{2}=\frac{\left(1-\lambda_{0}|z|^{2}\right)^{2}}{\left(1-\lambda|f|^{2}\right)^{2}}\left|\frac{\mathrm{d} f}{\mathrm{~d} z}\right|^{2},
$$


where $z$ is a local complex coordinate on $M_{0}$. Notice that the norm of the local section (Higgs field) is given by a Jacobian of the coordinate transformation between the Riemann surfaces $M_{0}$ and $M$, multiplied by the holomorphic tangent of the function $f$. Vortices are thus ramification points of the auxiliary map $f$. It is also known from Ref. [12] that all these exotic vortex equations can be reached by dimensional reduction of $G$-instantons from the four manifold $M_{0} \times \Sigma$ for an appropriate choice of gauge group $G$ and Riemann surface $\Sigma$. For the well known hyperbolic case $G=\mathrm{SU}(2)$ and $\Sigma=S^{2}$ while for Popov vortices $G=\mathrm{SU}(1,1)$ and $\Sigma=\mathbb{H}^{2}$.

Following Ref. [10] the potential energy piece of the Ginzburg-Landau action functional, adapted to the $\left(\lambda_{0}, \lambda\right)$ vortex, is given by

$$
V=\frac{1}{2} \int_{M_{0}}\left(\frac{1}{\Omega_{0}^{2}}\left(F_{12}\right)^{2}+\frac{2 \lambda}{\Omega_{0}}\left|D_{i} \phi\right|^{2}+\left(\lambda_{0}-\lambda|\phi|^{2}\right)^{2}\right) \Omega_{0} \mathrm{~d}^{2} x, \quad i=1,2,
$$

where $F_{12}=-2 \mathrm{i} F_{z \bar{z}}$ and

$$
\mathrm{d} \phi-\mathrm{i} A \phi=D_{i} \phi \mathrm{d} x^{i}, \quad i=1,2 .
$$

A Bogomol'nyi type argument on this functional gives rise to the vortex equations (2.1),

$$
V=\frac{1}{2} \int_{M_{0}}\left[\left(\frac{F_{12}}{\Omega_{0}}-\lambda_{0}+\lambda|\phi|^{2}\right)^{2}+\frac{8 \lambda}{\Omega_{0}}\left|D_{\bar{z}} \phi\right|^{2}\right] \Omega_{0} \mathrm{~d}^{2} x+2 \pi \lambda_{0} k,
$$

where we have dropped a boundary term and defined the magnetic flux

$$
k:=\frac{1}{2 \pi} \int_{M_{0}} F=\frac{1}{2 \pi} \int_{M_{0}} F_{12} \mathrm{~d}^{2} x .
$$

The Bogomol'nyi bound is then

$$
V \geq V_{\mathrm{BPS}}=2 \pi \lambda_{0} k
$$

where BPS stands for Bogomol'nyi-Prasad-Sommerfield.

\section{Magnetic impurities}

Magnetic impurities are an important but also a simple type of impurity found in materials, like superconductors. In Ref. [13] vortices in the presence of a magnetic impurity, $\sigma(x)$, were considered and the latter is taken into account by simply changing the potential term as

$$
\frac{1}{2}\left(\lambda_{0}-\lambda|\phi|^{2}\right)^{2} \quad \longrightarrow \quad \frac{1}{2}\left(\lambda_{0}-\lambda|\phi|^{2}-\sigma(x)\right)^{2}
$$

as well introducing a static source for the magnetic field

$$
L_{\text {impurity }}=2 \int_{M_{0}} \sigma(x) F_{12} \mathrm{~d}^{2} x .
$$

Note that the magnetic field, $B$, is given by $B=\Omega_{0}^{-1} F_{12}$ and hence the conformal factor $\Omega_{0}$ cancels out in the source term for the impurity. 
A Bogomol'nyi argument still works and results now in the first-order vortex equations

$$
(\mathrm{d} \phi-\mathrm{i} A \phi) \wedge e=0, \quad F=\mathrm{d} A=\left(\lambda_{0}-\lambda|\phi|^{2}-\sigma(x)\right) \omega_{0},
$$

or in terms of local coordinates

$$
\begin{aligned}
\partial_{\bar{z}} \phi-\mathrm{i} A_{\bar{z}} \phi & =0 \\
F_{z \bar{z}}=\partial_{z} A_{\bar{z}}-\partial_{\bar{z}} A_{z} & =\frac{\mathrm{i}}{2} \Omega_{0}\left(\lambda_{0}-\lambda|\phi|^{2}-\sigma(z, \bar{z})\right) .
\end{aligned}
$$

In Ref. [14], the case of Taubes vortices, $\left(\lambda_{0}, \lambda\right)=(1,1)$, was explored and the effect of the impurity on the moduli space metric was considered. In particular, if the integrable case of Taubes vortices on the hyperbolic disk is taken, then for a delta function defect the vortex equations are still integrable. We shall see shortly that this is also the case for the other integrable $\left(\lambda_{0}, \lambda\right)$ vortex equations.

\subsection{Exotic Liouville-type equations with impurities}

Applying the standard decomposition of $\phi=e^{h+\mathrm{i} \chi}$ and using the first vortex equation (3.4) to solve for $A$ we can reduce Eqs. (3.4)-(3.5) to a single equation for $h$ of Liouville-type:

$$
-\frac{4}{\Omega_{0}} \partial_{z} \partial_{\bar{z}} h=\left(\lambda_{0}-\lambda e^{2 h}-\sigma(z, \bar{z})\right)-\frac{2 \pi}{\Omega_{0}} \sum_{r=1}^{N} \delta\left(z-Z_{r}\right),
$$

where the delta function sources are at the points $Z_{r}$ and correspond to the zeros of $\phi$.

We take the conformal factor to be

$$
\Omega_{0}=\frac{4}{\left(1-\lambda_{0}|z|^{2}\right)^{2}},
$$

that is, $M_{0}$ is a Riemann surface with constant curvature $K_{0}=-\lambda_{0}$. For the case of $\mathbb{H}^{2}$ and $\mathbb{S}^{2}$ this conformal factor corresponds to a disk of radius 1 and a sphere of radius 1 , respectively.

Following the approach in Ref. [8], we make the substitution

$$
h=g+\log \left(\frac{1}{2}\left(1-\lambda_{0}|z|^{2}\right)\right)
$$

such that

$$
4 \partial_{z} \partial_{\bar{z}} h=4 \partial_{z} \partial_{\bar{z}} g-\lambda_{0} \Omega_{0} .
$$

This transforms the equation for $h$ into

$$
4 \partial_{z} \partial_{\bar{z}} g=\lambda e^{2 g}+\Omega_{0} \sigma+2 \pi \sum_{r=1}^{N} \delta\left(z-Z_{r}\right),
$$

with $K=-\lambda$ being the Gauss curvature of $M$ (away from the points $Z_{r}$ which correspond to the zeros of $\phi$ ).

There are three cases where this reduces to Liouville's equation. 
- The first is when the defect is zero, $\sigma=0$, as then Eq. (3.10) becomes [10]

$$
4 \partial_{z} \partial_{\bar{z}} g=\lambda e^{2 g}+2 \pi \sum_{r=1}^{N} \delta\left(z-Z_{r}\right)
$$

which has the solution

$$
g=-\log \frac{1-\lambda|f|^{2}}{2}+\frac{1}{2} \log \left|\frac{\mathrm{d} f}{\mathrm{~d} z}\right|^{2}
$$

and leads to $[10]$

$$
\phi=\frac{1-\lambda_{0}|z|^{2}}{1-\lambda|f|^{2}} \frac{\mathrm{d} f}{\mathrm{~d} z}, \quad A_{\bar{z}}=-\mathrm{i} \partial_{\bar{z}} \log \left(\frac{1-\lambda_{0}|z|^{2}}{1-\lambda|f|^{2}}\right),
$$

in the unitary gauge.

- The second case is when the defect is given in terms of delta functions

$$
\sigma=\frac{2 \pi}{\Omega_{0}} \sum_{j=1}^{K} \alpha_{j} \delta\left(z-Z_{N+j}\right), \quad \alpha_{j} \in \mathbb{R}_{>0},
$$

as then Eq. (3.10) becomes

$$
4 \partial_{z} \partial_{\bar{z}} g=\lambda e^{2 g}+2 \pi \sum_{j=1}^{K} \alpha_{j} \delta\left(z-Z_{N+j}\right)+2 \pi \sum_{r=1}^{N} \delta\left(z-Z_{r}\right) .
$$

Only when $\alpha_{j} \in \mathbb{Z}_{>0}$ are positive integers, the impurities have the interpretation of being sources for vortices. If $\alpha_{j}:=1, \forall j$, we have

$$
4 \partial_{z} \partial_{\bar{z}} g=\lambda e^{2 g}+2 \pi \sum_{r=1}^{N+K} \delta\left(z-Z_{r}\right),
$$

which is still completely general because $\left\{Z_{r}\right\}$ are allowed to be coincident.

In Ref. [14] it was pointed out that the defect being a delta function does not make sense in the Lagrangian where a square of a delta function would appear, but the Eqs. (3.5) and (3.10) are still sensible to consider. It is also important to note that we want $\phi$ to be an $N$ vortex solution however, Eq. (3.16) would naturally lead to an $N+K$ vortex solution.

Consider the case $K=1, \alpha:=\alpha_{1} \in \mathbb{R}_{>0}$ a positive real constant but not necessarily an integer, set $Z_{N+1}:=0$, and denote by $f(z):=z \tilde{f}(z)$ a holomorphic function with $N$ ramification points, which enters the solution $g$ of Eq. (3.12) to the impurity-less Taubes Eq. (3.11). Then we have that $f(z):=z^{\alpha+1} \tilde{f}(z)$, plugged into $g$ of Eq. (3.12), is a solution to the Taubes equation with the impurity (and general $\alpha \in \mathbb{R}_{>0}$ ) (3.15). The Higgs field corresponding to $g$ with $f(z):=z^{\alpha+1} \tilde{f}(z)$ reads

$$
\phi=\frac{1-\lambda_{0}|z|^{2}}{1-\lambda|z|^{2 \alpha+2}|\tilde{f}|^{2}}\left((\alpha+1) z^{\alpha} \tilde{f}+z^{\alpha+1} \frac{\mathrm{d} \tilde{f}}{\mathrm{~d} z}\right),
$$


in a certain choice of gauge. As pointed out in Ref. [14] for hyperbolic vortices (i.e. $(1,1)$ vortices), this actually looks like a vortex with winding number $N+\alpha$ with $\phi$ multivalued when $\alpha \notin \mathbb{Z}$. The singular gauge transformation

$$
\phi \rightarrow \sqrt{\frac{\bar{z}^{\alpha}}{z^{\alpha}}} \phi=\frac{|z|^{\alpha}}{z^{\alpha}} \phi
$$

is needed to make this look like a $N$ vortex. $^{2}$

- The final case where we return to Liouville's equation and integrability, is when the defect parameter is a constant. Then we have Liouville's equation for a surface with Gauss curvature $\lambda_{0}-\sigma$. The same scaling argument as invoked in Ref. [10] and mentioned above means that we have to consider only three cases, $\lambda_{0}-\sigma=-1,0,1$. This means that a constant defect enables integrable vortices to be constructed on a surface with the "wrong" Gauss curvature. This relates Popov, Jackiw-Pi, and Ambjørn-Olesen vortices. ${ }^{3}$

\section{Product gauge groups}

In Ref. [13] the authors showed how, in the case of Taubes vortices, the impurity term could be viewed as being due to a heavy frozen vortex coupled to a different gauge group. Their method describes a coupled gauge theory with two scalar fields, charged under two gauge groups. In particular, one scalar field is in the bifundamental representation, while the other is in the fundamental representation of the second gauge group. This can be generalized to arbitrarily charged scalar fields and extends to $\left(\lambda_{0}, \lambda\right)$ vortices as we now show.

We will follow the notation of Ref. [16] and consider the $\mathrm{U}(1)^{2}$ gauge theory with two Higgs fields $\phi_{A}, A=1,2$, where the two gauge fields $A_{i}^{a}$ belong to $\mathrm{U}(1)_{a}, a=1,2 . A$ is the flavor index, $a$ is the gauge group index and finally, $i=1,2$ is the spatial index of vector fields. The charges of the Higgs fields are contained in the charge matrix $Q$, specified by the gauge covariant derivative

$$
D_{i} \phi_{A} \mathrm{~d} x^{i}=\mathrm{d} \phi_{A}-\mathrm{i} \sum_{a} Q_{A a} A^{a} \phi_{A},
$$

with $A^{a}=A_{i}^{a} \mathrm{~d} x^{i}$ being the two 1 -forms belonging to the gauge groups $\mathrm{U}(1)_{a}, a=1,2$, respectively. We will not assume a nonvanishing determinant of the charge matrix, as was done in Ref. [16], since it will be an unnecessary assumption in what follows. If on the other hand, one restricts to the case $\operatorname{det} Q \neq 0$, the non-integrable system of hyperbolic vortex equations reduces to that studied in Ref. [22]. Note that we have absorbed the gauge coupling $e$ by changing length scales in this paper, which in particle physics would make all elements of $Q$ integers (or for quarks, multiples of $1 / 6$ ). In this paper, we will allow the elements of $Q$ to take on any real values.

\footnotetext{
${ }^{2}$ This will shove the multivalued $\alpha$ phase of the Higgs field into the gauge field.

${ }^{3}$ Of course physically, it is semantics whether the VEV $\lambda_{0}$ is physically the VEV or it has further contributions from other "fields", like $\lambda_{0}-\sigma$, when $\sigma$ is a constant.
} 
The field strengths corresponding to the two gauge groups are given by

$$
F^{a}=\mathrm{d} A^{a}=\left(\partial_{1} A_{2}^{a}-\partial_{2} A_{1}^{a}\right) \mathrm{d} x^{1} \wedge \mathrm{d} x^{2} .
$$

The static (potential) energy of the theory is

$$
V=\frac{1}{2} \int_{M_{0}}\left[\frac{1}{\Omega_{0}^{2}} \sum_{a}\left(F_{12}^{a}\right)^{2}+\frac{2 \lambda}{\Omega_{0}} \sum_{A}\left|D_{i} \phi_{A}\right|^{2}+\sum_{a}\left(\lambda \sum_{A}\left|\phi_{A}\right|^{2} Q_{A a}-\lambda_{0} r_{a}\right)^{2}\right] \Omega_{0} \mathrm{~d}^{2} x
$$

where $r_{a}, a=1,2$, are real constants. The vacuum of the theory is

$$
\lambda \sum_{A}\left|\phi_{A}\right|^{2} Q_{A a}=\lambda_{0} r_{a}
$$

which we require to hold for every $a$, putting certain conditions on $r_{a}$; if the $\left\{r_{a}\right\}$ 's do not satisfy the above equation, this is known as supersymmetry breaking (D-term breaking) in physics. Note that we have left an ambiguity of the sign of the constant terms in $\lambda_{0}$, but $r_{a} \in \mathbb{R}$ are kept as real numbers for now, which makes it possible to have different magnitudes or signs in the two resulting vortex equations.

A Bogomol'nyi trick can readily be performed

$$
\begin{aligned}
V= & \frac{1}{2} \int_{M_{0}}\left[\sum_{a}\left(\frac{F_{12}^{a}}{\Omega_{0}}-\lambda_{0} r_{a}+\lambda \sum_{A}\left|\phi_{A}\right|^{2} Q_{A a}\right)^{2}+\frac{8 \lambda}{\Omega_{0}} \sum_{A}\left|D_{\bar{z}} \phi_{A}\right|^{2}\right] \Omega_{0} \mathrm{~d}^{2} x \\
& +2 \pi \lambda_{0} \sum_{a} r_{a} k^{a}
\end{aligned}
$$

where we have defined the magnetic fluxes

$$
k^{a}:=\frac{1}{2 \pi} \int_{M_{0}} F^{a}=\frac{1}{2 \pi} \int_{M_{0}} F_{12}^{a} \mathrm{~d}^{2} x, \quad a=1,2 .
$$

Note that the magnetic fluxes may not be integers in this model. It is now easy to read off the Bogomol'nyi equations

$$
\begin{aligned}
D_{\bar{z}} \phi_{A} & =0 \\
\frac{1}{\Omega_{0}} F_{12}^{a} & =\lambda_{0} r_{a}-\lambda \sum_{A}\left|\phi_{A}\right|^{2} Q_{A a} .
\end{aligned}
$$

When the above equations are satisfied, the Bogomol'nyi bound is saturated

$$
V \geq V_{\mathrm{BPS}}=2 \pi \lambda_{0} \sum_{a} r_{a} k^{a} .
$$

The first Bogomol'nyi equation can be solved by the gauge fields

$$
\sum_{a} Q_{A a} A_{\bar{z}}^{a}=-\mathrm{i} \partial_{\bar{z}} \log \phi_{A},
$$


from which $\sum_{a} Q_{A a} F_{12}^{a}$ can be calculated, yielding

$$
-\frac{2}{\Omega_{0}} \partial_{z} \partial_{\bar{z}} \log \left|\phi_{A}\right|^{2}=\lambda_{0} \sum_{a} Q_{A a} r_{a}-\lambda \sum_{a, B} Q_{A a} Q_{B a}\left|\phi_{B}\right|^{2}-\frac{2 \pi}{\Omega_{0}} \sum_{r=1}^{N_{A}} \delta\left(z-Z_{r}^{A}\right),
$$

where we have multiplied the second Bogomol'nyi equation by $Q_{A a}$ and summed over $a$ and $N_{A}$ is the number of vortices (zeros) in the $A$ th flavor, counted with multiplicity. The winding number or the vortex number, $N_{A}$, is given by the number of zeros in $\phi_{A}$, counted with multiplicity.

The winding number of the $A$-th flavor of Higgs field, $N_{A}$, is linearly related to the fluxes $k^{a}$ by the charge matrix. This can be seen by using $\sum_{a} Q_{A a} F_{12}^{a}$ in Eq. (4.6), yielding

$$
\sum_{a} Q_{A a} k^{a}=-\frac{1}{\pi} \int_{M_{0}} \partial_{z} \partial_{\bar{z}} \log \left|\phi_{A}\right|^{2} \mathrm{~d}^{2} x=-\frac{1}{2 \pi \mathrm{i}} \oint_{\partial M_{0}} \partial_{z} \log \left|\phi_{A}\right|^{2} \mathrm{~d} z
$$

Using that at the boundary, the Higgs field tends to $\phi_{A} \propto z^{N_{A}}$,

$$
\sum_{a} Q_{A a} k^{a}=-N_{A}
$$

we obtain the relation between the magnetic fluxes $k^{a}$ and the winding number $N_{A}$, as promised. The fluxes $k^{a}$ correspond to the degrees of the line bundles over $M_{0}$ with connections $A^{a}$ and smooth sections $\phi_{A}$. For a nondiagonal charge matrix the sections are linearly mixed according to the above equation. Note that although the winding numbers, $N_{A} \in \mathbb{Z}_{>0}$, are positive integers, which is necessary for the complex scalar fields to be single valued (and vortices as opposed to anti-vortices), the magnetic fluxes $k_{a}$ need not be integers - even if all the components of the charge matrix are integers.

Using the standard decomposition $\phi_{A}=e^{h_{A}+\mathrm{i} \chi_{A}}$, we arrive at

$$
-\frac{4}{\Omega_{0}} \partial_{z} \partial_{\bar{z}} h_{A}=\lambda_{0} \sum_{a} Q_{A a} r_{a}-\lambda \sum_{a, B} Q_{A a} Q_{B a} e^{2 h_{B}}-\frac{2 \pi}{\Omega_{0}} \sum_{r=1}^{N_{A}} \delta\left(z-Z_{r}^{A}\right) .
$$

Changing variables from $h_{A}$ to $g_{A}$ by

$$
h_{A}=g_{A}+\log \frac{1-\lambda_{0} \sum_{a} Q_{A a} r_{a}|z|^{2}}{2},
$$

we get

$$
4 \partial_{z} \partial_{\bar{z}} g_{A}=\lambda \sum_{a, B} Q_{A a} Q_{B a} e^{2 g_{B}}+2 \pi \sum_{r=1}^{N_{A}} \delta\left(z-Z_{r}^{A}\right)
$$

provided that

$$
\sum_{a} Q_{A a} r_{a}=1,
$$

holds for all $A=1,2$. This condition is the compatibility condition for the coupled Liouville-type equations with respect to the background geometry of the Riemann manifold $M_{0}$. 
Note that by imposing the above geometric compatibility condition, $h_{A}$ of eq. (4.15) reduces to

$$
h_{A}=g_{A}+\log \left(\Omega_{0}^{-\frac{1}{2}}\right)
$$

Explicitly, this means that we only get the coupled vortex equations (4.16) for a Riemann surface of constant Gauss curvature $K_{0}=-\lambda_{0}$.

\subsection{Toda system}

In the particular case where the charge matrix satisfies

$$
\sum_{a} Q_{A a} Q_{B a}=K_{A B}=\left(\begin{array}{cc}
2 & -1 \\
-1 & 2
\end{array}\right)
$$

where $K_{A B}$ is the $\mathrm{SU}(3)$ Cartan matrix, then the coupled Liouville-type equations reduce to the integrable Toda system ${ }^{4}$. There is a 1-parameter family of solutions to this equation, given by

$$
Q=\left(\begin{array}{c}
\mp^{\prime} \frac{\sqrt{2-d^{2}}}{2} \mp^{\prime} \frac{ \pm \sqrt{3} d}{2}-\frac{d}{2} \pm \frac{\sqrt{3}}{2} \sqrt{2-d^{2}} \\
\pm^{\prime} \sqrt{2-d^{2}}
\end{array}\right), \quad d \in[-\sqrt{2}, \sqrt{2}]
$$

where \pm and $\pm^{\prime}$ are two independent signs and $Q Q^{\mathrm{T}}=K$. This solution for the charge matrix has

$$
\operatorname{det} Q=\mp^{\prime} \pm \sqrt{\operatorname{det} K}=\mp^{\prime} \pm \sqrt{3}
$$

for any value of $d \in[-\sqrt{2}, \sqrt{2}]$. As a specific example, we may choose $d:=0$ yielding

$$
Q=\frac{1}{\sqrt{2}}\left(\begin{array}{cc}
\mp^{\prime} 1 & \pm \sqrt{3} \\
\pm^{\prime} 2 & 0
\end{array}\right)=\frac{1}{\sqrt{2}}\left(\begin{array}{cc}
-1 & \sqrt{3} \\
2 & 0
\end{array}\right)
$$

where we have chosen the upper signs on the right-hand side. The Fayet-Iliopoulos parameters

$$
\left(\begin{array}{l}
r_{1} \\
r_{2}
\end{array}\right)=\frac{1}{2}\left(\begin{array}{c}
\mp^{\prime} \pm \sqrt{3} d \pm^{\prime} \sqrt{2-d^{2}} \\
\pm \sqrt{3\left(2-d^{2}\right)}+d
\end{array}\right)
$$

are a solution to the geometric compatibility condition (4.17) and for the charge matrix (4.22) corresponding to $d:=0$, they reduce to

$$
\left(\begin{array}{l}
r_{1} \\
r_{2}
\end{array}\right)=\frac{1}{\sqrt{2}}\left(\begin{array}{c} 
\pm^{\prime} 1 \\
\pm \sqrt{3}
\end{array}\right)=\frac{1}{\sqrt{2}}\left(\begin{array}{c}
1 \\
\sqrt{3}
\end{array}\right)
$$

where we have chosen the upper signs on the right-hand side.

\footnotetext{
${ }^{4}$ Toda equations occur when $K$ is the Cartan matrix of any simple Lie algebra. Thus there will also be Toda equations corresponding to the Cartan matrix for $G_{2}$.
} 
We thus arrive at the system of equations

$$
4 \partial_{z} \partial_{\bar{z}} g_{A}=\lambda \sum_{B} K_{A B} e^{2 g_{B}}+2 \pi \sum_{r=1}^{N_{A}} \delta\left(z-Z_{r}^{A}\right),
$$

with $K_{A B}$ given in Eq. (4.19) and $A, B=1,2$. If we set $\lambda:=-1$, which corresponds to the coupled Popov, coupled Jackiw-Pi or coupled Ambjørn-Olesen vortices, this is exactly the Toda system

$$
4 \partial_{z} \partial_{\bar{z}} g_{A}=-\sum_{B} K_{A B} e^{2 g_{B}}+2 \pi \sum_{r=1}^{N_{A}} \delta\left(z-Z_{r}^{A}\right) .
$$

The Toda system of equations is known to be integrable and for $K_{A B}$ of Eq. (4.19), the solution of the above system of equations is given in terms of the Kostant-Leznov-Saveliev solution [17-19], which is given by

$$
\exp \left(2 g_{A}\right)=\frac{1}{2} 4 \partial_{z} \partial_{\bar{z}} \log \operatorname{det}\left(M_{A}^{\dagger} M_{A}\right),
$$

( $A$ not summed over) where $M_{A}$ is a 3 -by- $A$ rectangular matrix. Since we only have two flavors $(A=1,2)$, the matrices $M_{A}$ in our case are given by ${ }^{5}$

$$
M_{1}=u, \quad M_{2}=\left(u, \partial_{z} u\right),
$$

with $u(z)$ the holomorphic 3 -vector

$$
u:=\left(\begin{array}{c}
1 \\
f_{1}(z) \\
f_{2}(z)
\end{array}\right) .
$$

Here the 3 -vector is due to the Toda system being the $\mathrm{SU}(3)$ case, which has the Cartan matrix given in Eq. (4.19). The ramification points of $f_{1,2}(z)$ correspond to vortex positions.

We can generalize the Kostant-Leznov-Saveliev solution to both signs of $\lambda= \pm 1$, by introducing the sign in the solution as follows

$$
\exp \left(2 g_{A}\right)=-\frac{1}{2 \lambda} 4 \partial_{z} \partial_{\bar{z}} \log \operatorname{det}\left(M_{A}^{\dagger} W_{A}\right),
$$

( $A$ not summed over) where $M_{A}$ and $W_{A}$ are 3 -by- $A$ rectangular matrices. For the two flavors $(A=1,2)$, the matrices $M_{A}, W_{A}$ in our case are given by ${ }^{6}$

$$
M_{1}=u, \quad M_{2}=\left(u, \partial_{z} u\right), \quad W_{1}=v, \quad W_{2}=\left(v, \partial_{z} v\right)
$$

\footnotetext{
${ }^{5}$ In the general case of the Toda system with a rank $R$ Lie algebra, the matrices are $(R+1)$-by- $A$ rectangular matrices given by $M_{A}:=\left(u, \partial_{z} u, \partial_{z}^{2} u, \cdots, \partial_{z}^{A-1} u\right)$ and $u$ is given by $\left(1, f_{1}(z), f_{2}(z), \cdots, f_{R}(z)\right)^{\mathrm{T}}$, where $A=1,2, \ldots, R$.

${ }^{6}$ In the general case of the Toda system with a rank $R$ Lie algebra, the matrices are $(R+1)$-by- $A$ rectangular matrices given by $M_{A}:=\left(u, \partial_{z} u, \partial_{z}^{2} u, \cdots, \partial_{z}^{A-1} u\right)$ and $W_{A}:=\left(v, \partial_{z} v, \partial_{z}^{2} v, \cdots, \partial_{z}^{A-1} v\right)$ with $u$ given by $\left(1, f_{1}(z), f_{2}(z), \cdots, f_{R}(z)\right)^{\mathrm{T}}$ and $v$ given by $\left(1,-\lambda f_{1}(z),-\lambda f_{2}(z), \cdots,-\lambda f_{R}(z)\right)^{\mathrm{T}}$, where $A=$ $1,2, \ldots, R$.
} 
with $u(z)$ and $v(z)$ the holomorphic 3-vectors

$$
u:=\left(\begin{array}{c}
1 \\
f_{1}(z) \\
f_{2}(z)
\end{array}\right), \quad v:=\left(\begin{array}{c}
1 \\
-\lambda f_{1}(z) \\
-\lambda f_{2}(z)
\end{array}\right) .
$$

For $\lambda=-1$, the original Kostant-Leznov-Saveliev solution is recovered.

What about the $\lambda=0$ case? Since there is no mixing between the two vortex equations for $\lambda=0$, the solution is simply the standard integrable Bradlow vortex solution of Ref. [10] in each field, as reviewed in Sec. 2 .

Remarkably, we have succeeded in constructing an integrable (2-by-2) vortex system of equations for any of the $\left(\lambda_{0}, \lambda\right)$ vortices, provided the geometric compatibility condition (4.17) is satisfied. What the condition imposes is to ensure that both vortex flavors can get the Fayet-Iliopoulos constant absorbed by the same background geometry.

\subsection{Impurities from product gauge groups}

Returning to the general product gauge group setup. Let us do a partial Bogomol'nyi trick on the energy functional (4.3), say on the second scalar field $\phi_{2}$ and the second gauge group $\mathrm{U}(1)_{2}$ :

$$
\begin{aligned}
V=\frac{1}{2} \int_{M_{0}}[ & \left(\frac{F_{12}^{2}}{\Omega_{0}}-\lambda_{0} r_{2}+\lambda \sum_{A}\left|\phi_{A}\right|^{2} Q_{A 2}\right)^{2}+\frac{8 \lambda}{\Omega_{0}}\left|D_{\bar{z}} \phi_{2}\right|^{2}+2 \lambda_{0} r_{2} \frac{F_{12}^{2}}{\Omega_{0}} \\
& -\frac{2 \lambda}{\Omega_{0}} F_{12}^{2}\left|\phi_{1}\right|^{2} Q_{12}+\frac{2 \lambda}{\Omega_{0}} F_{12}^{1}\left|\phi_{2}\right|^{2} Q_{21} \\
& \left.+\frac{1}{\Omega_{0}^{2}}\left(F_{12}^{1}\right)^{2}+\left(-\lambda_{0} r_{1}+\lambda \sum_{A}\left|\phi_{A}\right|^{2} Q_{A 1}\right)^{2}+\frac{2 \lambda}{\Omega_{0}}\left|D_{i} \phi_{1}\right|^{2}\right] \Omega_{0} \mathrm{~d}^{2} x
\end{aligned}
$$

where the third line contains the terms not included in the Bogomol'nyi trick and the first line looks like a normal Bogomol'nyi completion. However, due to the non-diagonal charge matrix, there is a mismatch of two terms on the second line. So far, everything is symmetric, but to facilitate the discussion of the magnetic-type impurity interpretation of Ref. [13], we set

$$
Q_{21}:=0
$$

which reduces the above energy functional to

$$
\begin{aligned}
V=\frac{1}{2} \int_{M_{0}}[ & \left(\frac{F_{12}^{2}}{\Omega_{0}}-\lambda_{0} r_{2}+\lambda\left|\phi_{1}\right|^{2} Q_{12}+\lambda\left|\phi_{2}\right|^{2} Q_{22}\right)^{2}+\frac{8 \lambda}{\Omega_{0}}\left|\left(\partial_{\bar{z}}-\mathrm{i} Q_{22} A_{\bar{z}}^{2}\right) \phi_{2}\right|^{2}+2 \lambda_{0} r_{2} \frac{F_{12}^{2}}{\Omega_{0}} \\
& -\frac{2 \lambda}{\Omega_{0}} F_{12}^{2}\left|\phi_{1}\right|^{2} Q_{12} \\
& \left.+\frac{1}{\Omega_{0}^{2}}\left(F_{12}^{1}\right)^{2}+\left(\lambda_{0} r_{1}-\lambda\left|\phi_{1}\right|^{2} Q_{11}\right)^{2}+\frac{2 \lambda}{\Omega_{0}}\left|\left(\partial_{i}-\mathrm{i} Q_{11} A_{i}^{1}-\mathrm{i} Q_{12} A_{i}^{2}\right) \phi_{1}\right|^{2}\right] \Omega_{0} \mathrm{~d}^{2} x .
\end{aligned}
$$


We will now assume that the two vortex equations, corresponding to the two first positive definite terms, are satisfied and thus set them to zero. The second term, corresponding to $D_{\bar{z}} \phi_{2}=0$, determines $\phi_{2}$ in terms of the gauge field $A^{2}$. However, the vortex equation corresponding to the first term is coupled to $\phi_{1}$, so the magnetic flux $\frac{F_{12}^{2}}{\Omega_{0}}$ depends on $\phi_{1}$ and hence so does $\phi_{2}$. The last term on the first line is a topological invariant (counting the number of zeros in the field $\phi_{2}$ ) and is hence a constant in the energy.

It will now be convenient to redefine (diagonalize) the gauge field for $\phi_{1}$ as

$$
Q_{11} \mathcal{A}:=Q_{11} A^{1}+Q_{12} A^{2} \quad \Rightarrow \quad Q_{11} \mathcal{F}=Q_{11} F^{1}+Q_{12} F^{2} .
$$

Eliminating $F_{12}^{1}$ and imposing the vortex equations, we arrive at

$$
\begin{aligned}
V= & \frac{1}{2} \int_{M_{0}}\left[\frac{1}{\Omega_{0}^{2}}\left(\mathcal{F}_{12}\right)^{2}+\frac{2 \lambda}{\Omega_{0}}\left|D_{i}^{\mathcal{A}} \phi_{1}\right|^{2}+\left(\lambda_{0} r_{1}-\lambda\left|\phi_{1}\right|^{2} Q_{11}-\sigma(x)\right)^{2}+\frac{2}{\Omega_{0}} \sigma(x) \mathcal{F}_{12}\right] \Omega_{0} \mathrm{~d}^{2} x \\
& +2 \pi \lambda_{0}\left(r_{2}-\frac{Q_{12}}{Q_{11}} r_{1}\right) k^{2},
\end{aligned}
$$

where we have defined

$$
\begin{aligned}
D_{i}^{\mathcal{A}} & :=\partial_{i}-\mathrm{i} Q_{11} \mathcal{A}_{i}, \\
\sigma(x) & :=-\frac{Q_{12} F_{12}^{2}}{Q_{11} \Omega_{0}},
\end{aligned}
$$

with $k^{2}$ the topological degree of the vortex background (i.e. number of vortices in $\phi_{2}$ multiplied by $\left.-1 / Q_{22}\right)$.

The Bogomol'nyi trick on the first flavor of Higgs fields now leads to the vortex equations with impurities following Ref. [13]

$$
V=\frac{1}{2} \int_{M_{0}}\left[\left(\frac{\mathcal{F}_{12}}{\Omega_{0}}-\lambda_{0} r_{1}+\lambda\left|\phi_{1}\right|^{2} Q_{11}-\sigma(x)\right)^{2}+\frac{8 \lambda}{\Omega_{0}}\left|D_{\bar{z}}^{\mathcal{A}} \phi_{1}\right|^{2}\right] \Omega_{0} \mathrm{~d}^{2} x+2 \pi \lambda_{0} r_{1} k,
$$

where we have dropped the constant $\propto k^{2}$ and defined

$$
k:=\frac{1}{2 \pi} \int_{M_{0}} \mathcal{F}=\frac{1}{2 \pi} \int_{M_{0}} \mathcal{F}_{12} \mathrm{~d}^{2} x
$$

The Bogomol'nyi bound is thus

$$
V \geq V_{\mathrm{BPS}}=2 \pi \lambda_{0} r_{1} k
$$

We have thus generalized the Tong-Wong construction [13] of coupled vortex equations as an interpretation of the magnetic impurities, to the full five exotic $\left(\lambda_{0}, \lambda\right)$ vortex equations of Ref. [10] and additionally with generic charge matrix $Q$ of the form

$$
Q_{A a}=\left(\begin{array}{cc}
Q_{11} & Q_{12} \\
0 & Q_{22}
\end{array}\right)_{A a}
$$


where the rows are flavors of Higgs field and the columns are the gauge groups. The imposition of the vortex equations in Eq. (4.35) is thought of as freezing one species of vortices and this is what gives rise to the impurities for the effective theory with only the other vortex species left.

In the special case of

$$
Q_{A a}=\left(\begin{array}{cc}
1 & -1 \\
0 & 1
\end{array}\right),
$$

the two Higgs fields have the interpretation of a bifundamental field $\left(\phi_{1}\right)$ and a fundamental field $\left(\phi_{2}\right)$ of the second gauge group.

\subsubsection{What became of the topological charge?}

In Sec. 3.1, we discussed the situation of the impurities being delta functions and seemingly increasing the number of vortices in the remaining Higgs field (here $\phi_{1}$ ). What is the interpretation in the coupled vortex system? - We can now see more clearly what is happening, because the magnetic flux $k$ is really a linear combination of the degrees of the two line bundles. Writing out the Bogomol'nyi mass in terms of the original degrees $k^{1}, k^{2}$, we have

$$
V_{\mathrm{BPS}}=2 \pi \lambda_{0} r_{1}\left(k^{1}+\frac{Q_{12}}{Q_{11}} k^{2}\right) .
$$

Remembering now that we discarded a constant proportional to $k^{2}$, the total Bogomol'nyi mass is actually

$$
\begin{aligned}
V_{\mathrm{BPS}} & =2 \pi \lambda_{0} r_{1}\left(k^{1}+\frac{Q_{12}}{Q_{11}} k^{2}\right)+2 \pi \lambda_{0}\left(r_{2}-\frac{Q_{12}}{Q_{11}} r_{1}\right) k^{2} \\
& =2 \pi \lambda_{0}\left(r_{1} k^{1}+r_{2} k^{2}\right),
\end{aligned}
$$

and we are back to the total energy being the sum of the degrees of each fiber. The deceiving appearances of an extra topological charge from the impurity are simply due to the change of variables. We recall that the degrees $k^{a}$ are not necessarily integers for arbitrary charge matrices.

\subsection{General coupled vortex equations}

If we consider the general case of the vortex equations (4.14) without imposing the geometric compatibility condition (4.17), the system of equations is not integrable. In this section, we consider a specific family of examples and calculate numerical solutions. Here we keep $\left(\lambda_{0}, \lambda\right)$ general, but fix the charge matrix to that of Eq. (4.44), for which the system of vortex equations reduces to

$$
\begin{aligned}
& -\frac{4}{\Omega_{0}} \partial_{z} \partial_{\bar{z}} h_{1}=\lambda_{0}\left(r_{1}-r_{2}\right)-\lambda\left(2 e^{2 h_{1}}-e^{2 h_{2}}\right)-\frac{2 \pi}{\Omega_{0}} \sum_{r=1}^{N_{1}} \delta\left(z-Z_{r}^{1}\right), \\
& -\frac{4}{\Omega_{0}} \partial_{z} \partial_{\bar{z}} h_{2}=\lambda_{0} r_{2}-\lambda\left(-e^{2 h_{1}}+e^{2 h_{2}}\right)-\frac{2 \pi}{\Omega_{0}} \sum_{r=1}^{N_{2}} \delta\left(z-Z_{r}^{2}\right) .
\end{aligned}
$$


Choosing the constants to be $r_{1}=r_{2}=1$, we arrive at

$$
\begin{aligned}
& -\frac{4}{\Omega_{0}} \partial_{z} \partial_{\bar{z}} h_{1}=-\lambda\left(2 e^{2 h_{1}}-e^{2 h_{2}}\right)-\frac{2 \pi}{\Omega_{0}} \sum_{r=1}^{N_{1}} \delta\left(z-Z_{r}^{1}\right), \\
& -\frac{4}{\Omega_{0}} \partial_{z} \partial_{\bar{z}} h_{2}=\lambda_{0}-\lambda\left(-e^{2 h_{1}}+e^{2 h_{2}}\right)-\frac{2 \pi}{\Omega_{0}} \sum_{r=1}^{N_{2}} \delta\left(z-Z_{r}^{2}\right) .
\end{aligned}
$$
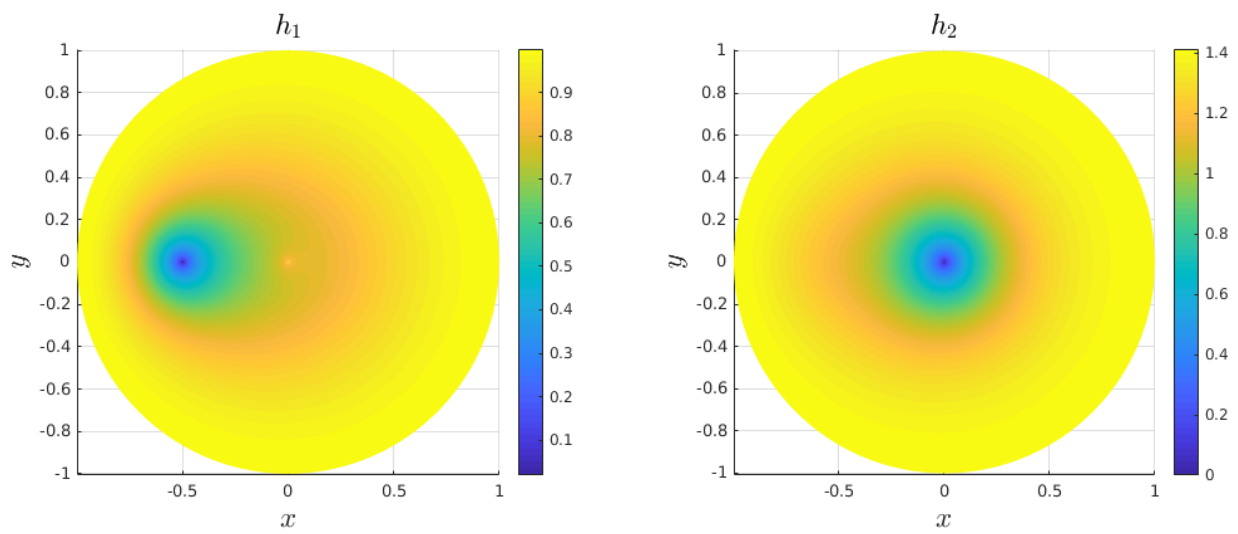

Figure 1. A numerical solution of the $(1,1)$ coupled vortices on the Poincare disk model of $\mathbb{H}^{2}$. The vortex centers are given in Eq. (4.51). The left-hand side panel, showing the field $h_{1}$, can be interpreted as a vortex in the presence of an impurity sitting at the center of the disk.

As an example, we calculate a numerical solution to Eqs. (4.49)-(4.50) in the $(1,1)$ case, corresponding to the coupled Taubes equations with the following vortex positions

$$
Z_{1}^{1}=-\frac{1}{2}, \quad Z_{1}^{2}=0
$$

The result is shown in Fig. 1. In the context of freezing the second vortex field $h_{2}$, as was done in Sec. 4.2, the first vortex $h_{1}$ can be interpreted as a single vortex experiencing an impurity sitting at the origin of the disk.

\section{Discussion and conclusion}

In this paper, we have considered the five "exotic" vortex equations studied by Manton in Ref. [10] and generalized all of them to include magnetic impurities, first put forward for Taubes equations on $\mathbb{R}^{2}$ by Tong and Wong in Ref. [13]. The integrability properties considered in Ref. [14] in the case of Taubes $\left(\lambda_{0}, \lambda\right)=(1,1)$ vortices carry over to all five exotic vortex equations. In particular, a delta function impurity does not break integrability and looks like a source of extra vorticity. This has a simple interpretation when the impurity is formulated as a second vortex field in a product gauge group theory, as was considered already for Taubes vortices in Ref. [13]. For the product gauge group theory, we furthermore generalize the theory to encompass a generic charge matrix following Ref. [16]. We do not need to impose that the charge matrix is invertible, as was assumed for the 
analysis in Ref. [16], but certain constraints on the Fayet-Iliopoulos parameters must hold for the vacuum to exist. First we write down the generic case of $\left(\lambda_{0}, \lambda\right)$ vortices with generic charges and generic Fayet-Iliopoulos parameters. In order to arrive at a coupled Liouville-type equation, hoping to obtain the Toda system of equations, we find a geometric compatibility condition that is a condition on the charge matrix and the Fayet-Iliopoulos parameters. A specific choice of charge matrix reduces the system to the SU(3) Toda equation, which for $\lambda=-1$ is solved by the renowned Kostant-Leznov-Saveliev solution [17-19]. We further generalize this solution to the $\lambda=1$ case by a suitable modification of the Kostant-Leznov-Saveliev solution. This solution is to the best of our knowledge new. The $\lambda=0$ case is solved by the Bradlow solution of Ref. [10] in each field. This gives in total five exotic integrable Toda vortex solutions for Hyperbolic-Toda, Popov-Toda, Jackiw-Pi-Toda, Ambjørn-Olesen-Toda and Bradlow-Toda equations ${ }^{7}$. Then we show that the product gauge group theory generalizes the argument of Tong and Wong [13] that a second frozen vortex is interpreted as an impurity of the first species of vortex; our generalization extends to all five vortex equations and generic charge matrices of uppertriangular form. Finally, we construct a numerical solution in the case of coupled hyperbolic $\left(\lambda_{0}, \lambda\right)=(1,1)$ vortex equations and the interpretation of the impurity is visualized.

Our construction can trivially be extended to $N$ coupled vortex equations in a $\mathrm{U}(1)^{N}$ gauge theory. All the results carry over by simply extending the range of the indices, except for the case of the impurities. In that case, one would have to decide how many flavors of Higgs fields are being frozen and how many remain. Such extension should be straightforward.

One could further consider if there are some natural geometric interpretations that could be made for some aspects of our construction, for instance related to the impurities. That is, do the integrable cases of the coupled vortex equations admit a Baptista type geometric interpretation where the vortices give rise to a degenerate metric [11]? Another interesting question is whether there exist further possibilities of integrable solutions involving an impurity in either of the vortex equations.

\section{Acknowledgments}

S. B. G. thanks the Outstanding Talent Program of Henan University for partial support. The work of S. B. G. is supported by the National Natural Science Foundation of China (Grants No. 11675223 and No. 12071111).

C. R. thanks Steffen Krusch for introducing him to the topic of vortices with magnetic impurities.

\footnotetext{
${ }^{7}$ The latter is not really a Toda system of equations, because $\lambda=0$ eliminates the mixing of the fields.
} 


\section{References}

[1] C. H. Taubes, Arbitrary N: Vortex Solutions to the First Order Landau-Ginzburg Equations, Commun. Math. Phys. 72, 277-292 (1980).

[2] N. S. Manton and P. M. Sutcliffe, Topological solitons, Cambridge monographs on mathematical physics, Cambridge University Press, 2004.

[3] A. Jaffe and C. Taubes, Vortices and monopoles: Structure of static gauge theories, Progress in physics, Birkhäuser Boston, 1980.

[4] Y. Yisong, Solitons in Field Theory and Nonlinear Analysis, Springer monographs in Mathematics, Springer, 2001.

[5] R. Jackiw and S. Y. Pi, Soliton Solutions to the Gauged Nonlinear Schrodinger Equation on the Plane, Phys. Rev. Lett. 64, 2969-2972 (1990).

[6] R. Jackiw and S. Y. Pi, Classical and quantal nonrelativistic Chern-Simons theory, Phys. Rev. D 42, 3500 (1990) [erratum: Phys. Rev. D 48, 3929 (1993)].

[7] J. Ambjorn and P. Olesen, Antiscreening of Large Magnetic Fields by Vector Bosons, Phys. Lett. B 214, 565-569 (1988).

[8] E. Witten, Some Exact Multi-Instanton Solutions of Classical Yang-Mills Theory, Phys. Rev. Lett. 38, 121-124 (1977).

[9] A. D. Popov, Integrable vortex-type equations on the two-sphere, Phys. Rev. D 86, 105044 (2012) [arXiv:1208.3578 [hep-th]].

[10] N. S. Manton, Five Vortex Equations, J. Phys. A 50, no.12, 125403 (2017) [arXiv:1612.06710 [hep-th]].

[11] J. M. Baptista, Vortices as degenerate metrics, Lett. Math. Phys. 104, 731-747 (2014) [arXiv:1212.3561 [hep-th]].

[12] F. Contatto and M. Dunajski, Manton's five vortex equations from self-duality, J. Phys. A 50, no.37, 375201 (2017) [arXiv:1704.05875 [hep-th]].

[13] D. Tong and K. Wong, Vortices and Impurities, JHEP 01, 090 (2014) [arXiv:1309.2644 [hep-th]].

[14] A. Cockburn, S. Krusch and A. A. Muhamed, Dynamics of vortices with magnetic impurities, J. Math. Phys. 58, no.6, 063509 (2017) [arXiv:1512.01054 [hep-th]].

[15] C. Adam, T. Romanczukiewicz and A. Wereszczynski, The $\phi^{4}$ model with the BPS preserving defect, JHEP 03, 131 (2019) [arXiv:1812.04007 [hep-th]].

[16] S. B. Gudnason, M. Eto and M. Nitta, 1/2-BPS vortex strings in $\mathcal{N}=2$ supersymmetric $\mathrm{U}(1)^{N}$ gauge theories, J. Math. Phys. 62, no.3, 032304 (2021) [arXiv:2008.13440 [hep-th]].

[17] B. Kostant, The Solution to a generalized Toda lattice and representation theory, Adv. Math. 34, 195-338 (1979).

[18] A. N. Leznov and M. V. Saveliev, Representation of zero curvature for the system of nonlinear partial differential equations $x_{\alpha, z \bar{z}}=\exp (k x)_{\alpha}$ and its integrability, Lett. Math. Phys. 3, 489-494 (1979).

[19] A. N. Leznov, On Complete Integrability of a System of Nonlinear Partial Differential Equations in Two-dimensional Space, Theor. Math. Phys. 42, 225 (1980).

[20] N. S. Manton, Vortex solutions of the Popov equations, J. Phys. A 46, 145402 (2013) 
[arXiv:1211.4352 [hep-th]].

[21] S. B. Gudnason and M. Nitta, Some exact Bradlow vortex solutions, JHEP 05, 039 (2017) [arXiv:1701.04356 [hep-th]].

[22] X. Han and Y. Yang, Magnetic Impurity Inspired Abelian Higgs Vortices, JHEP 02, 046 (2016) [arXiv:1510.07077 [hep-th]]. 